\title{
Covariation in conditioned response strength between stimuli trained in compound
}

\author{
LOUIS D. MATZEL, KARL SHUSTER, and RALPH R. MILLER \\ State University of New York, Binghamton, New York
}

\begin{abstract}
Conditioned lick suppression by water-deprived rats was used to elaborate on recent evidence that the attenuated conditioned response elicited by an overshadowed stimulus may be enhanced by extinction of the overshadowing stimulus with which it had been trained in simultaneous compound. Using a modified serial stimulus arrangement in which a light coexisted with the last half of a tone that terminated with footshock, it was found in Experiment 1 that the tone overshadowed the light. Extinction of the tone-shock association resulted in a virtually complete recovery of the response to the overshadowed light. Using this serial overshadowing procedure, the possibility that the strength of a conditioned response to an element trained in compound covaries as a function of the strength of the response to the other element was tested in Experiment 2. Following overshadowing training similar to that of Experiment 1, independent reinforcement of the overshadowed light, that is, associative inflation, was found to have no deleterious effect on the response to the overshadowing tone. This suggests that the effects of postconditioning extinction and inflation of one element do not have symmetrical effects upon responding to the other element. The results of Experiment 2 were replicated in Experiment 3 using a simultaneous compound stimulus as opposed to the serial compound of the previous studies. These results are discussed in terms of various associative and cognitive models of learning and performance.
\end{abstract}

Recent reports by Kaufman and Bolles (1981) and Matzel, Schachtman, and Miller (1985) have indicated that the attenuated response evoked by a weak stimulus (X) following reinforcement in compound with a more salient stimulus (A) can be enhanced by postconditioning extinction (i.e., nonreinforced exposure) of $A$. These results challenge the traditional view that "overshadowing" deficits represent an acquisition failure with respect to the less salient cue (Kamin, 1969a; Mackintosh, 1975; Pavlov, 1927; Rescorla \& Wagner, 1972). Kamin, for instance, suggested that when the more salient A stimulus is reinforced in compound with $\mathrm{X}, \mathrm{A}$ acquires associative strength at a more rapid rate, quickly becoming a reliable predictor of the unconditioned stimulus (US). As the US is no longer "surprising," the less salient $X$ cue, which is still at a subasymptotic associative level, acquires no additional associative strength. Similarly, Rescorla and Wagner (1972) proposed that the associative strength that a given US presentation can support is limited, and the more salient cue in a reinforced compound accrues the available strength at the expense of the less salient cue. These views of overshadowing assume that increments in associative strength are determined by the subject's expectation of the particular US paired with the compound stimulus. In contrast, both Mackintosh (1975) and Pearce

\footnotetext{
Support for this research was provided by NSF Grant 86-00755. We are grateful to Ronald Sigmundi for his helpful comments on an early draft of this report. Address reprint requests to Ralph R. Miller, Department of Psychology, SUNY-Binghamton, Binghamton, NY 13901.
}

and Hall (1980) argued that the overshadowing deficit represents a failure to process the less salient cue during compound conditioning.

The common assumption of all of these models is that overshadowing represents a failure by the less salient cue to accrue associative strength, and hence cannot account for the enhanced conditioned response evoked by that stimulus following extinction of the overshadowing cue (Kaufman \& Bolles, 1981; Matzel et al., 1985). Matzel et al. tested several possible interpretations of this effect. For example, Kasprow, Cacheiro, Balaz, and Miller (1982) reported that brief nonreinforced exposure to an overshadowed cue in some instances enhanced the response to that cue. This observation led them to conclude that overshadowing represented, at least in part, a retrieval failure. However, Matzel et al. found no evidence to support the possibility that extinction exposure to A serves to facilitate later retrieval of the X-US association, or that simultaneous reactivation of the $X$ and US representations during the extinction of A actually enhances the X-US association. Moreover, they determined that the facilitated response to the overshadowed stimulus is specific to the extinction of the overshadowing stimulus as opposed to the extinction of any other excitatory cue previously trained with the same US. These results led Matzel et al. to suggest that the response evoked by the overshadowed cue covaries with the response evoked by the overshadowing cue.

In an attempt to account for similar findings, Kaufman and Bolles (1981) proposed that both elements of a reinforced compound become associated with the US, but 
that, in the case of overshadowing, the X-US association is not expressed in behavior provided that the A-US association is sufficient to fulfill the animal's attribution of cause. Extinction of the overshadowing A stimulus "disconfirms" this expectancy, allowing the X-US association to be manifest in behavior. Alternatively stated, the reinforced compound stimulus presentations may provide the animal with ambiguous information, and subsequent extinction of an individual element may result in a reassessment of probable cause.

In a somewhat less cognitive interpretation of conditioned response generation, Miller and Schachtman (1985) suggested that the conditioned response evoked by a particular conditioned stimulus (CS) represents a comparison, performed at the time of testing, of the associative strength of that stimulus to the associative strength of stimuli that were present in close proximity to the CS during training. Although evidence to support this claim has focused on variations in associative strength of the CS relative to its training context, this comparator hypothesis may also apply to overshadowing, with the more salient overshadowing cue serving as part of the comparator term for the less salient overshadowed cue (and vice versa). Consequently, postconditioning extinction of the overshadowing cue might facilitate the response to the overshadowed cue, just as Miller and Schachtman found that postconditioning extinction of an excitatory context enhances responding to a CS trained in that context.

In the present series of studies, we examined whether the results of Kaufman and Bolles (1981) and Matzel et al. (1985) would generalize to serial overshadowing, and also tested a prediction implied by Kaufman and Bolles and by Miller and Schachtman (1985). Specifically, in their most elemental forms, Kaufman and Bolles's and Miller and Schachtman's models suggest that although each stimulus of a reinforced compound may be associated with the US, the response evoked by either element is inversely related to the associative strength of the alternate element at the time of testing. Hence, postconditioning extinction of one element should enhance the conditioned response (CR) to the alternate element, and independent reinforcement of an element following compound training should attenuate responding to the alternate element. However, it should be noted that, although Miller and Schachtman (1985) found that postconditioning extinction of the conditioning context produces enhanced responding to a CS trained in that context (paralleling Kaufman \& Bolles's and Matzel et al.'s finding with two discrete CSs), postconditioning associative inflation of the conditioning context fails to impair responding to the CS, even when testing occurs in a neutral context. These results led Miller and Schachtman to conclude that associative extinction and inflation of one element of a compound may not have symmetrical effects upon responding to the other element of the compound (see, also, Kaplan \& Hearst, 1985, for similar results and conclusions).

\section{EXPERIMENT 1}

Experiment 1 was intended to establish an overshadowing preparation in which extinction of the overshadowing stimulus resulted in a fully restored response to the overshadowed cue. Our previous research on simultaneous overshadowing (Matzel et al., 1985) had consistently found less than complete recovery of the response to the overshadowed stimulus with the particular parameters used. The present study employed a modified serial overshadowing procedure. This procedure has two possible advantages over the previous work. First, the onset of the overshadowed stimulus in the serial case bears better temporal contiguity to the US than does the overshadowing cue, which might enhance the response to the overshadowed cue following extinction of the overshadowing stimulus. Second, in the serial case, the two elements of the compound are not coextensive. Such an arrangement would be expected to reduce any perceptual configuring of the elements (Wasserman, Carr, \& Deich, 1978), which might mitigate against a recovery of the response to the overshadowed stimulus invoked by extinction of the overshadowing stimulus.

With conditioned lick suppression as the dependent variable, a 5-sec flashing light overlapped the last half of a 10 -sec tone. The offset of the tone-light compound was reinforced with a footshock US. Relative to a control group trained in the absence of the tone, the light trained in the quasi-serial compound evoked an attenuated conditioned response. Subsequently, we attempted to eliminate this performance deficit by extinguishing the toneshock association.

\section{Method}

Subjects. Twelve male and 12 female naive Sprague-Dawleydescended rats obtained from our own breeding colony served as subjects. The rats ranged in age from 80 to 90 days at the onset of the experiment. Body weights were $260-380 \mathrm{~g}$ for males and $210-280 \mathrm{~g}$ for females. The animals were individually housed in standard hanging wire-mesh cages in a vivarium maintained on a 16-h/8-h light/dark cycle, with experimental manipulations occurring during the middle portion of the light phase of the cycle. The animals were allowed free access to food, but water availability was limited to $10 \mathrm{~min} /$ day following a progressive deprivation schedule imposed 6 days prior to initiation of the study.

Apparatus. Twelve chambers, measuring $24.1 \times 12.7 \times 20 \mathrm{~cm}$ $(1 \times w \times h)$ and housed in individual environmental isolation chests, were used. Three of the chamber side walls were opaque Plexiglas; the ceiling and remaining side wall were clear Plexiglas. Diffused illumination was provided in each chamber by a 1.12-W bulb located behind one opaque wall. Chamber floors consisted of parallel stainless steel rods that were interconnected by NE-2 neon bulbs in series with a 1-M $\Omega$ resistor. The output from a high-voltage ac power source could be passed through this circuit to provide a 1.0-mA constant-current footshock. When an animal bridged a neon bulb, the animal's resistance decreased to approximately $20 \mathrm{k} \Omega$ and the bulb's resistance rose to several megohms, thereby effectively shunting all the current through the subject. Each chamber was equipped with a water-filled lick tube that extended through a 
horizontal slit in one side wall $2.5 \mathrm{~cm}$ above the floor. By licking the tube during sessions in which shock was not administered, the animal completed a circuit between the water and the grid floor, which allowed the number of licks to be monitored. Nominal CSs consisted of a normally off 1.12-W overhead light, which when activated, flashed $0.5 \mathrm{sec}$ on $/ 0.5 \mathrm{sec}$ off, and a $1000-\mathrm{Hz}$ tone delivered by a $45-\Omega$ overhead speaker. This auditory CS was $12 \mathrm{~dB}(\mathrm{C})$, re SPL, above an $80-\mathrm{dB}(\mathrm{C})$ background noise level produced by a ventilation fan mounted on the side of each environmental chest.

Procedure. On Days 1-4, all animals received one $30-\mathrm{min}$ session/day in the conditioning enclosure. On Day 1, the lick tube aperture extended $1 \mathrm{~cm}$ into the chamber; on all subsequent days, it was flush with the chamber wall. These sessions allowed the animals to acclimate to the conditioning enclosures and to establish baseline lick rates. Latencies to complete the first and second 25 licks were recorded on each day for all subjects. As there were no appreciable differences across animals for either measure on Day 4 , the subjects were assigned randomly to treatment conditions, with the restriction that the groups be counterbalanced to the extent possible for body weight, litter, sex, and Day 4 latencies for the second 25 licks.

Conditioning was conducted on Days 5 and 6. On each of these days, all animals were placed in the chambers for 60 min during which they received four reinforced trials. Group $\mathrm{O}$ (overshadowing, $n=8$ ) received four $10-$ sec presentations of the tone. Five seconds after onset of the tone, the flashing light was activated for the remaining $5 \mathrm{sec}$ of each tone presentation. The stimulus complex was reinforced at offset with a $1-\mathrm{sec}$ footshock. Instead of the compound stimulus, Group OC (overshadowing control, $n=8$ ) received a 5-sec presentation of the flashing light alone, which was reinforced at offset with the 1-sec footshock. In addition, Group OC received four nonreinforced 10 -sec tone-alone presentations, which were interspersed among the reinforced trials. Group $\mathrm{O} / \mathrm{T}-$ (overshadowing, tone extinction, $n=8$ ) received conditioning treatments identical to those of Group $O$. Reinforced trials for all groups occurred $15,26,36$, and 52 min into the 60 -min session on the first day of conditioning and $5,16,30$, and 42 min into the session on the second day. Tone presentations for Group OC occurred 10, 22, 33 , and 48 min into each session. Conditioning was conducted on baseline, that is, with the lick tubes present.

On Days 7-10, Group O/T- received 12 10-sec nonreinforced exposures/day to the tone alone (extinction), randomly distributed throughout a 90 -min session in the conditioning chambers. Groups $O$ and $O C$ were also placed in the conditioning chambers for $90 \mathrm{~min}$, but received no tone presentations. Lick tubes were present for all groups.

Testing was conducted on Days 11 and 12. On Day 11, all animals were placed in the conditioning chambers and were allowed to complete 25 licks. Immediately following the 25 th lick, the flashing light (overshadowed stimulus) was presented and maintained until the subject completed an additional 25 licks. Thus, all animals were drinking at the onset of the test stimulus. After completion of the second 25 licks, the animal was removed from the chamber and returned to the home cage. On Day 12 , an identical test was conducted with the tone CS. A 1,200-sec ceiling was applied to test scores. In practice, no animals in this or the subsequent studies reached this ceiling.

\section{Results and Discussion}

Latencies to complete Licks 0-25 and Licks 25-50 were transformed to log latencies in order to permit the use of parametric statistics. One subject in Group O/T - received incorrect treatment and was eliminated from the study. There were no significant between-group differences in the first 25 licks on either test day ( $p s>.25$ ). All means were less than $1.0 \mathrm{log} \mathrm{sec}$.
Figure 1 illustrates the mean latency for each group to complete 25 licks in the presence of both the light and tone. A one-factor analysis of variance (ANOVA) on the response to the light indicated a significant effect of treatment $[F(2,20)=4.14, p<.03]$. Individual groups were contrasted with planned comparisons. Testing on the light revealed overshadowing of the light by the tone, as indicated by a comparison of Groups $O$ and $\operatorname{OC}[F(1,20)=$ $6.87, p<.02$ ]. Extinction of the tone resulted in a recovery from this performance deficit, that is, Group O/Texhibited more suppression to the light than did Group $\mathrm{O}$ $[F(1,20)=5.72, p<.03]$ but did not differ from Group OC $[F(1,20)<1]$.

Conditioned responding to the overshadowing tone was significantly greater in Group $\mathrm{O}$ than in both Group OC $[F(1,20)=19.33, p<.01]$ and Group $\mathrm{O} / \mathrm{T}-[F(1,20)$ $=19.27, p<.01]$, indicating associative acquisition in Group $O$ relative to Group $O C$ and subsequent extinction of that association in Group $\mathrm{O} / \mathrm{T}-$. No difference in response to the tone existed between Groups $\mathrm{O} / \mathrm{T}-$ and OC $[F(1,20)<1]$.

Collectively, these results indicate overshadowing of the light by the tone. This overshadowing presumably was due to the temporal arrangement of the two stimuli rather than to differences in their independent saliences (Experiment 3 indicated that these stimuli were of roughly equal salience, as measured by their acquisition rates when conditioned with equal presentation durations). Although additional control conditions would have delineated the relative contributions of these two possible sources of overshadowing, they were not included because this issue was not of central interest here.

Beyond the observation of overshadowing, Experiment 1 determined that extinction of the overshadowing tone resulted in a virtually complete recovery of the response to the otherwise overshadowed light (see Fig-

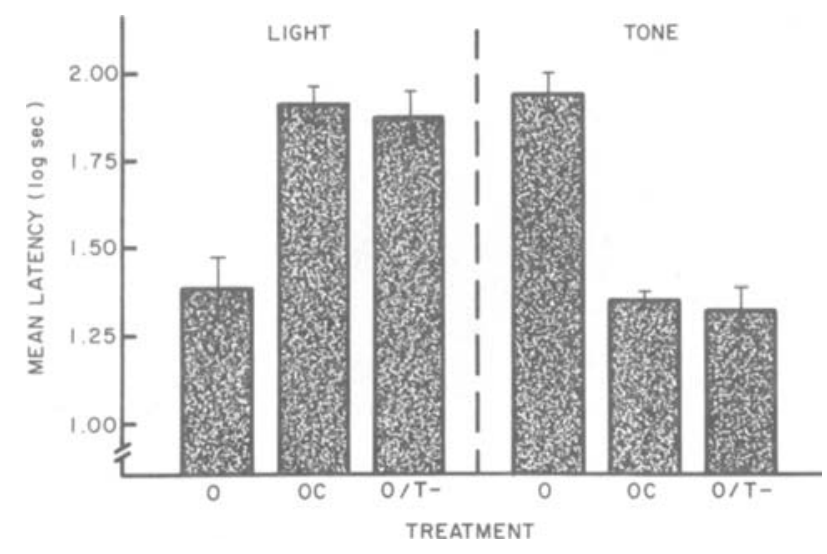

Figure 1. Mean latencies on the test days to complete 25 licks in the presence of the light (overshadowed stimulus) and tone (overshadowing stimulus). $\mathrm{O}=$ overshadowing (tone + light serial compound paired with shock); $\mathrm{OC}=$ overshadowing control (light paired with shock); $\mathrm{O} / \mathrm{T}=$ overshadowing, tone extinction (tone + light serial compound paired with shock followed by tone extinction trials). Brackets indicate standard errors. 
ure 1). In previous work, using the more traditional procedure in which the overshadowed and overshadowing stimulus were trained in simultaneous compound, Matzel et al. (1985) reported only a moderate recovery from overshadowing. Two possibilities could account for the more complete recovery observed here. Due to the independent onset of the tone and light, the animal is more likely to have processed the light and tone as individual elements as opposed to a single perceptual unit. If such configuring were to occur, it would be expected to result in a loss of associative responding to an element if the other element was extinguished (Rescorla, 1981; Speers, Gillan, \& Rescorla, 1980). That extinction of one element resulted in an increase in the CR to the alternate element in the present study argues against the occurrence of perceptual configuring under the present conditions. In addition, the serial arrangement provided a 5-sec delay between light and US onsets as opposed to a 10-sec delay between the tone onset and the US, which might be expected to support a stronger light-US association even though not manifest in behavior as long as the tone was the most valid predictor of the US.

In a previous report on serial overshadowing, Egger and Miller (1962) suggested that a second (overshadowed) stimulus in a compound is redundant and hence the animal fails to associate it with the US. Moreover, Egger and Miller found that occasional nonreinforced presentations of the overshadowing cue interspersed among the initial compound conditioning trials result in an attenuation of overshadowing, presumably owing to an increase in the relative validity of the otherwise redundant stimulus (also see Wagner, 1969). The current results suggest that the associative strength of the overshadowing stimulus (i.e., the tone) at the time of compound training is not sufficient to account for the retarded response to the overshadowed cue (i.e., the light). Rather, the response evoked by the light appears to be inversely related to the response evoked by the overshadowing stimulus at the time of testing.

\section{EXPERIMENT 2}

The observation that the CR to one element of a previously reinforced compound stimulus increases as a result of extinction of the other element suggests that the inverse may also be true. That is, responding to one element may decrease as a result of further conditioning of the other element. This outcome (procedurally backward blocking) is predicted by the attributional analysis of Kaufman and Bolles (1981). In fact, Kamin (1969b) performed such a test and found a moderate loss of responding to a CS initially trained in compound when the alternate element was subsequently reinforced independently. However, Kamin's study was confounded by a longer training-to-test interval in the critical group than in the control conditions, leading Kamin to conclude that this "reverse blocking", effect was the result of forgetting. Moreover, Schweitzer and Green (1982) and Seraganian and vom Saal (1969) performed similar manipulations, but found no loss of responding to a stimulus used in compound training when the other element of the compound was subsequently reinforced in isolation. However, Johnson and Cumming (1968) did observe a response decrement to a compound element under similar circumstances, leaving the issue unresolved (but see Seraganian \& vom Saal for a possible confound in the Johnson \& Cumming study). To further examine the possibility that associative inflation of a stimulus element previously reinforced in compound will result in a loss of response strength to the alternate element of the compound, we employed, in Experiment 2, exactly the same serial overshadowing procedure that had yielded negative transfer of extinction in the previous study. In contrast with Experiment 1, following the compound conditioning trials the overshadowed light was reinforced in isolation for one group of animals, whereas the remaining groups received comparable reinforcement of an irrelevant stimulus. The critical measure was now the response elicited by the overshadowing tone. Of particular interest was whether reinforcement of the light in isolation would adversely affect the response to the tone with which it previously had been trained in compound.

\section{Method}

Subjects. Twelve male and 12 female rats, similar to those used in Experiment 1, served in this study. Body weights ranged from 280 to $375 \mathrm{~g}$ for males, and from 210 to $255 \mathrm{~g}$ for females. Housing and deprivation conditions were the same as in Experiment 1.

Apparatus and Procedure. The apparatus and stimulus parameters were identical to those of the previous study except for the addition of a third stimulus, specifically a click train produced by a $5 / \mathrm{sec}$ operation of a $24-\mathrm{V}$ ac relay attached to the outside of the rear Plexiglas wall of each chamber. The click was $10 \mathrm{~dB}(\mathrm{C})$ above background and was accompanied by a slight vibration of the chamber walls and floor. All animals were acclimated to the experimental enclosures on Days 1-4, as in the previous study. Also as in Experiment 1, two groups received serial-compound training with the tone-light stimulus complex during initial conditioning on Days 5 and 6 (Groups $\mathrm{O}$ and $\mathrm{O} / \mathrm{L}+, n s=8$ ). Group $\mathrm{OC}(n=8)$ was initially trained on the light alone and also given nonreinforced exposure to the tone in a manner identical to Group OC in Experiment 1.

Following this initial training, Group $\mathrm{O} / \mathrm{L}+$ received four lightshock pairings a day for 2 days in 60 -min sessions. The light and shock parameters were the same as those used in initial conditioning. Groups $O$ and $O C$ received similar treatment except that the click was substituted for the light. After completion of this inflation phase of the study, all animals received one 60-min session in the conditioning context on Day 9 in the absence of any discrete stimulus presentations in order to insure stable lick rates on subsequent test days. Testing was conducted as in Experiment 1, except that the tone served as the test stimulus on Day 10, the light on Day 11 , and finally the click on Day 12 .

\section{Results and Discussion}

No differences between groups in latencies to complete the first 25 licks were found on either test day ( $p s>.25$ ). All means were less than $1.0 \log$ sec.

Figure 2 illustrates the mean latencies to complete 25 licks in the presence of the tone, light, and click. With respect to responding to the overshadowed stimulus 


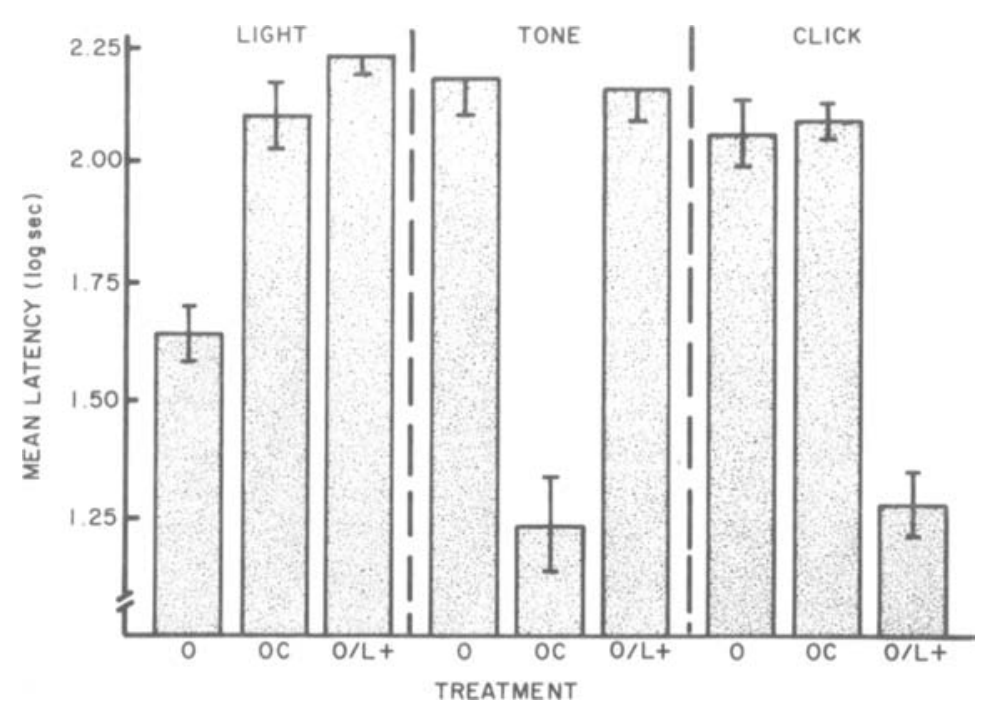

Figure 2. Mean latencies on the test days to complete 25 licks in the presence of the light (overshadowed stimulus), tone (overshadowing stimulus), and clicks (irrelevant conditioned excitor). $O=$ overshadowing (tone + light serial compound paired with shock); $\mathrm{OC}=$ overshadowing control (light paired with shock); $\mathrm{O} / \mathrm{L}+$ = overshadowing followed by light reinforcement (tone + light serial compound paired with shock followed by light-shock pairings). Brackets indicate standard errors.

(light), a one-factor ANOVA indicated a significant effect of treatment $[F(2,21)=7.57, p<.01]$. Planned comparisons found that Groups $\mathrm{OC}$ and $\mathrm{O} / \mathrm{L}+$ did not differ in response to the light $[F(1,21)=1.58, p>.20]$. As in Experiment 1, overshadowing of the light by the tone was evidenced in a comparison of Groups $\mathrm{O}$ and $\mathrm{OC}$ $[F(1,21)=6.75, p<.02]$. Groups $\mathrm{O}$ and $\mathrm{O} / \mathrm{L}+$ also differed $[F(1,21)=7.31, p<.02]$, indicating that independent reinforcement of the light following compound training did enhance the response to that stimulus.

Responding to the tone was of principal interest in the current study. A one-factor ANOVA indicated a significant effect of treatment $[F(2,21)=9.88, p<.01]$. Conditioned responding to the overshadowing tone was evident in Group O relative to Group OC $[F(1,21)=15.26$, $p<.01]$ and in Group $\mathrm{O} / \mathrm{L}+$ relative to Group OC $[F(1,21)=14.41, p<.01]$. Independent reinforcement of the overshadowed light had no deleterious effect on the response to the tone with which it had previously been reinforced in compound. This is indicated by the lack of difference between Groups $\mathrm{O}$ and $\mathrm{O} / \mathrm{L}+$ in response to the tone $[F(1,21)<1]$.

A one-factor ANOVA indicated that the response to the click stimulus was significantly greater in Groups $\mathbf{O}$ and OC, which had received eight reinforced click presentations on Days 7 and 8, than in Group $\mathrm{O} / \mathrm{L}+$, which had not received any click-shock pairings $[F(2,21)=17.84$, $p<.01]$.

These results suggest that the responses evoked by stimuli trained in compound do not covary in a simple fashion. Although Experiment 1 indicated that extinction (deflation) of the association to the overshadowing tone facili- tated responding to the overshadowed light, Experiment 2 found that independent reinforcement of the overshadowed light subsequent to compound stimulus training did not attenuate responding to the overshadowing tone.

\section{EXPERIMENT 3}

Experiments 1 and 2 found that extinction of an overshadowing cue can enhance responding to the overshadowed stimulus, but the symmetrical operation of independent reinforcement of the overshadowed stimulus apparently has no deleterious effect on responding to the overshadowing cue. However, this conclusion is possibly confounded by the lack of temporal symmetry between the light and tone in Experiments 1 and 2. During the initial overshadowing treatment in Experiments 1 and 2, the serial arrangement of cues permitted the onset of the tone to function as a signal for the onset of the light $5 \mathrm{sec}$ later. It is conceivable that in Experiment 2 the isolated reinforcement of the overshadowed light might have reduced the tone-shock association, but that responding to the tone may have been maintained through second-order associations to footshock mediated by the now excitatory light.

To eliminate this possible confound, we employed, in Experiment 3, a similar manipulation except that the stimuli were initially trained in simultaneous as opposed to serial compound. It should be remembered that Kaufman and Bolles (1981) and Matzel et al. (1985), using a simultaneous compound stimulus, found that, following reinforcement of the compound, extinction of one element enhanced responding to the other element. As in the previous studies, a light and a tone served as the initial 
compound training stimuli, but in this experiment they had simultaneous onsets and coterminated with shock. Since the light and tone were selected on the basis of being roughly equal in salience, no overshadowing was expected with this stimulus arrangement. Following compound conditioning, the light was reinforced in isolation for half of the animals; the remaining animals received comparable training with the click. The dependent measure of primary interest was the response to the tone as a function of the independent reinforcement of the light with which it had previously been reinforced in compound. These two groups were further divided into two subgroups. Half of the animals in each group were initially trained to a subasymptotic level (two pairings) on the tone-light compound in order to allow for observable associative inflation of the light and to minimize the possibility of a ceiling effect's obscuring any decrease in the associative strength of the tone. The remaining animals were initially trained to an asymptotic level (eight pairings) to protect against possible floor effects. If, as predicted by Kaufman and Bolles (1981), independent reinforcement of the light following compound training successfully degraded the response to the tone, the presence of both the two- and eight-pairing groups would allow us to determine if the effect was dependent on enhancement of the strength of responding to the light or merely on independent reinforcement of the light.

\section{Method}

Subjects. Twenty-four male and 24 female rats similar to those of Experiment 1 served in the present study. Body weights ranged from 275 to $390 \mathrm{~g}$ for males and from 200 to $270 \mathrm{~g}$ for females.
Housing and deprivation conditions were the same as in Experiment 1.

Apparatus and Procedure. The apparatus and stimulus parameters were identical to those of Experiment 2. The initial 4 days of the study served to acclimate the animals to the apparatus and establish a lick baseline, as in the previous studies. On Days 5 and 6, all subjects were conditioned with a 10 -sec stimulus consisting of the tone and light in simultaneous compound during a 60-min daily session. The offset of the stimulus compound was reinforced with footshock. Four groups of animals $(n s=12)$ received this treatment. Groups $2 / \mathrm{L}$ and $2 / \mathrm{C}$ received one such pairing in each of the two sessions for a total of two pairings. Groups $8 / \mathrm{L}$ and $8 / \mathrm{C}$ received four pairings in each session for a total of eight pairings.

On Days 7 and 8, all animals received additional conditioning with a single element during daily 60 -min sessions. Groups $2 / \mathrm{L}$ and $8 / \mathrm{L}$ received four light-shock pairings in each session, and Groups $2 / \mathrm{C}$ and $8 / \mathrm{C}$ received four click-shock pairings in each of these sessions. On the day following this treatment, all animals spent one 60-min session in the training enclosures in the absence of any discrete stimuli in order to ensure stable lick rates on the subsequent test days.

Testing of each stimulus was conducted as in Experiment 2.

\section{Results and Discussion}

Latencies to complete Licks 0-25 prior to presentation of test stimuli yielded no significant between-group differences on any test day $(p s>.25)$. All means were below 1.0 log sec.

Figure 3 illustrates the mean latency to complete 25 licks in the presence of the tone (Day 10), light (Day 11), and clicks (Day 12) for each group. First with respect to the light, a one-factor ANOVA revealed a significant effect of treatment $[F(3,44)=4.44, p<.01]$. As is evident from Figure 3, Group 2/C differed from the remain-

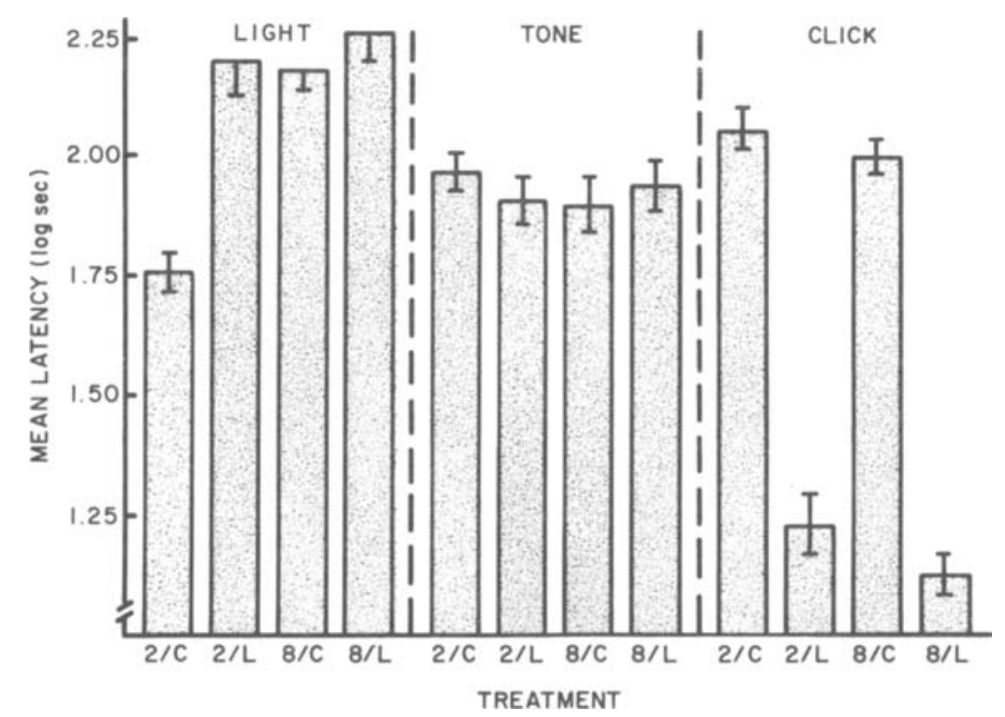

Figure 3. Mean latencies on test days to complete 25 licks in the presence of the light (overshadowed stimulus), tone (overshadowing stimulus), and click train (irrelevant conditioned excitor). All subjects received either two (2/C and $2 / \mathrm{L})$ or eight $(8 / \mathrm{C}$ and $8 / \mathrm{L}$ ) pairings of a simultaneous tone + light reinforced with shock. Subsequently, all animals received either click-shock pairings $(2 / \mathrm{C}$ and $8 / \mathrm{C})$ or light-shock pairings (2/L and $8 / L)$. Brackets indicate standard errors. 
ing groups $[F \mathrm{~s}(1,44) \geq 8.10, p \mathrm{~s}<.01]$. Groups $2 / \mathrm{L}$, $8 / \mathrm{C}$, and $8 / \mathrm{L}$ did not differ among themselves $\left[F_{\mathrm{s}}(1,44)<1\right]$. These results indicate that two light-tone compound trials were not sufficient for the light to evoke asymptotic suppression in Group $2 / \mathrm{C}$. The similarity of performance in Group 8/C (which received a total of eight reinforced presentations of the tone-light compound) and Group 8/L (which received eight initial reinforced compound pairings plus an additional eight reinforced presentations of the light alone) suggests that eight compound trials were sufficient to produce asymptotic suppression to the light. Thus, at least in Group $2 / \mathrm{L}$, the inflation manipulation following compound conditioning appears to have been successful in increasing suppression relative to its control, Group $2 / \mathrm{C}$. In contrast, the eight lightshock pairings given Group 8/L did not significantly increase suppression to the light in that group relative to Group 8/C. This suggests that an associative ceiling or possibly a subject-imposed performance ceiling was reached, although no animals in Group 8/L suppressed to the light for the maximum allowable $1,200 \mathrm{sec}$.

Next with respect to suppression to the tone, Figure 3 indicates similar levels of suppression across groups, which was confirmed by a one-factor ANOVA $[F(3,44)<1]$. This result implies that independent reinforcement of the light, even when accompanied by enhanced suppression to the light, did not result in a loss of suppression to the tone with which it was initially trained in simultaneous compound.

The responding to the click stimulus indicates significantly more suppression in Groups $2 / \mathrm{C}$ and $8 / \mathrm{C}$, both of which received eight reinforced click presentations, than in Groups $2 / \mathrm{L}$ and $8 / \mathrm{L}$, for which the click was associatively neutral at the time of testing $[F(3,44)=28.69$, $p<.01]$.

It is of interest to note that in Group $2 / \mathrm{C}$, although the light did not appear to reach an asymptotic associative level as the result of two reinforced presentations in compound with the tone, the tone apparently did. This is somewhat surprising, since the light apparently supported a higher asymptotic level of suppression than did the tone. In the language of Rescorla and Wagner (1972), the light seemingly had a higher $\lambda$ and a lower $\alpha$ than the tone. The asymptotic suppression evoked by the tone in Group 2/L was not likely due to an association between the tone and light that resulted in higher suppression to the tone following independent reinforcement of the light, since Group 2/C also exhibited asymptotic suppression to the tone without having received the light reinforced in isolation. A comparison of responding to the tone by Groups $2 / \mathrm{L}$ and $2 / \mathrm{C}$ does, in fact, suggest a lack of any within-compound association and/or configuring between the tone and light, since the two groups exhibit roughly equal suppression to the tone, even though Group $2 / \mathrm{L}$ received independent reinforcement of the light. However, this conclusion cannot be made with certainty, since any associative differences may be obscured by a subject- imposed performance ceiling, although none of the animals in Group 2/L suppressed to the tone for the maximum allowable time.

In conjunction with the findings of Experiments 1 and 2 , these results strengthen the conclusion that although extinction of a stimulus results in an enhanced CR to an alternate stimulus with which it was originally trained in compound, independent reinforcement of an element does not have a symmetrical effect, that is, it does not attenuate the response elicited by the other element.

\section{GENERAL DISCUSSION}

Numerous investigators (e.g., Balsam, 1985; Dickinson \& Charnock, 1985; Matzel, Brown, \& Miller, 1987) have reported that nonreinforced exposure to an excitatory context enhances the CR to a CS previously trained in that context. However, the inverse relationship has not typically been observed. For instance, Ayres and Benedict (1973), Kaplan and Hearst (1985), and Miller and Schachtman (1985) found no effect on the response to the target CS of postconditioning US-alone presentations in the context used for CS training, and Randich and Ross (1985) have reported that postconditioning US-alone presentations have only a small transitory attenuating effect on the response to an excitatory CS, an effect which they attribute to habituation. In short, convergent evidence indicates that although extinction of an excitatory association may enhance the excitatory potential of a stimulus trained in compound (simultaneous or successive) with the extinguished stimulus, inflation of a stimulus does not appear to attenuate the excitatory conditioned response to a stimulus trained in compound.

The present results have direct implications for several current theories of learning. The model of Rescorla and Wagner (1972) asserts that as the more salient cue in a stimulus compound reaches an associative asymptote, it blocks additional associative acquisition by the less salient cue, leaving it at a subasymptotic associative level. Likewise, Mackintosh (1975) and Pearce and Hall (1980) suggest that as the more salient CS becomes a good predictor of the US, attentional changes occur which decrease processing of the less salient cue. All of these theories assume that the less salient cue is not well associated with the US. If the redundancy of the second element in a serial compound results in reduced salience, the observations of Experiment 1 are inconsistent with the predictions of these models. Consistent with the prior results of Kaufman and Bolles (1981) and Matzel et al. (1985), extinction of the association between the overshadowing stimulus and the US facilitated the CR to the overshadowed stimulus. This result suggests that simple temporal contiguity may be sufficient to support associative learning, whereas expression of associations may be determined by response rules related to conventional views of contingency (see Matzel et al., 1987, for related results and discussion). 
Several recent hypotheses have suggested that acquisition is governed largely by contiguity and performance is related to contingency. Kaufman and Bolles (1981) proposed that causal attributions by the animal determine conditioned responding. Thus, an overshadowing stimulus is perceived as the cause of a US until it is extinguished, at which time causal attribution is redirected to the overshadowed stimulus, which was associated with the US, but without causal attribution, at the time of compound training. This prediction is consistent with the results of Experiment 1. However, implicit in the Kaufman and Bolles hypothesis is the symmetric prediction that inflation of one element of a two-element compound stimulus following reinforcement of the compound will attenuate responding to the other element. Both Experiments 2 and 3 of the present research and the studies cited above all failed to find such an effect.

The comparator hypothesis of Miller and Schachtman (1985) suggests that conditioned responding reflects a comparison of the associative strength of the CS to the associative strength of "comparator stimuli" that were temporally and spatially proximal to the CS during conditioning and include, but are not restricted to, the stimuli of the conditioning context. This comparison is said to occur at the time of testing. Thus, the comparator hypothesis in its simplest form predicts both a decrease and an increase in conditioned responding to the target CS when its comparator stimuli are associatively inflated or extinguished, respectively, following CS training. However, Miller and Schachtman were unable to find evidence that postconditioning inflation of the training context adversely affected the response to a stimulus trained in that context, leading them to suggest that the effects of postconditioning extinction and inflation are not symmetrical.

It can be concluded that no current model of associative learning and/or performance adequately describes the data presented here. Although Miller and Schachtman (1985) correctly anticipated a lack of response attenuation following the inflation manipulation employed here, this result is not a direct prediction of their model. Likewise, an attributional model such as that of Kaufman and Bolles (1981) predicts both the enhanced response to an element following extinction of the alternate element as well as an attenuated response following independent reinforcement of the alternate element. One might modify the attributional analysis by assuming that, in the animal's view, all events must have at least one cause but may have multiple causes. The problem with such an analysis is that it is vague regarding when an event will be attributed to a single cause and when it will be attributed to multiple causes given a compound CS, and in this simple form cannot easily describe overshadowing itself.

Lastly, it should be noted that the results reported in Experiment 1 would not be possible were the compound elements strongly associated and/or configured at the time of initial conditioning. In fact, Rescorla (1981), using parameters that favored within-compound associations and/or configuring, conducted experiments similar to those reported here and found that extinction and infla- tion of an element previously trained in compound resulted in an attenuation and enhancement, respectively, in the CR to the other element of the compound (see also Schweitzer \& Green, 1982). Several possibilities may account for the lack of an association between elements in the compound stimuli used in the current experiments. First, associations between stimuli appear to be reduced if the compound is reinforced (e.g., Holland, 1985). However, such associations are not necessarily eliminated, inasmuch as Rescorla (1981) found evidence of associations between compounded elements in an overshadowing paradigm. Rescorla's compound stimulus consisted of two flavors, which invites the suggestion that stimuli of different modalities such as those used here may be less disposed to form associations to one another (but see Cunningham, 1981). Moreover, the short stimulus durations employed here mitigate against the formation of within-compound associations (Rescorla, 1981). Also, it is likely that serial presentation of stimuli in Experiment 1 reduced any configuring of the stimulus complex; however, this possibility is not applicable to parallel results obtained by Matzel et al. (1985) and Kaufman and Bolles (1981) with a simultaneous stimulus arrangement.

\section{REFERENCES}

Ayres, J. J. B., \& BENEDICT, J. O. (1973). US-alone presentations as an extinction procedure. Animal Learning \& Behavior, 1, 5-8.

Balsam, P. D. (1985). The functions of context in learning and performance. In P. D. Balsam \& A. Tomie (Eds.), Context and learning (pp. 1-21). Hillsdale, NJ: Erlbaum.

Cunningham, C. L. (1981). Association between elements of a bivalent compound stimulus. Joumal of Experimental Psychology: Animal Behavior Processes, 7, 425-436.

Dickinson, A., \& CHARnock, D. J. (1985). Contingency effects with maintained instrumental reinforcement. Quarterly Journal of Experimental Psychology, 37B, 397-416.

EGger, M. D. , \& Miller, N. E. (1962). Secondary reinforcement in rats as a function of information value and reliability of the stimulus. Journal of Experimental Psychology, 64, 97-104.

HoLlAND, P. C. (1985). Element pretraining influences the content of appetitive serial compound conditioning in rats. Journal of Experimental Psychology: Animal Behavior Processes, 11, 367-387.

Johnson, D. F., \& Cumming, W. W. (1968). Some determiners of attention. Journal of the Experimental Analysis of Behavior, 11, 157-166.

Kamin, L. J. (1969a). Predictability, surprise, attention, and conditioning. In B. A. Cambell \& R. M. Church (Eds.), Punishment and aver sive behavior (pp. 279-296). New York: Appleton-Century-Crofts.

KAMIN, L. J. (1969b). Selective association and conditioning. In N. J. Mackintosh \& W. K. Honig (Eds.), Fundamental issues in associative learning (pp. 42-44). Halifax, NS, Canada: Dalhousie University Press.

KaPLAN, P. S., \& HeARst, E. (1985). Excitatory versus inhibitory learning: Studies of extinction, reinstatement, and interference. In P. D. Balsam \& A. Tomie (Eds.), Context and learning. Hillsdale, NJ: Erlbaum.

Kasprow, W. J., Cacheiro, H., Balaz, M. A., \& Miller, R. R. (1982). Reminder-induced recovery of associations to an overshadowed stimulus. Learning \& Motivation, 13, 155-166.

Kaufman, M. A., \& Bolles, R. C. (1981). A nonassociative aspect of overshadowing. Bulletin of the Psychonomic Society, 18, 318-320.

MaCkintosh, N. J. (1975). A theory of attention: Variations in the associability of stimuli with reinforcement. Psychological Review, 82 , 276-298.

Matzel, L. D., Brown, A. M., \& Miller, R. R. (1987). Associative 
effects of US preexposure: Retarded conditioned responding mediated by an excitatory training context. Joumal of Experimental $P_{s y}$ chology: Animal Behavior Processes, 13, 65-72.

Matzel, L. D., Schachtman, T. R., \& Miller, R. R. (1985). Recovery of an overshadowed association achieved by extinction of the overshadowing stimulus. Learning \& Motivation, 16, 398-412.

Miller, R. R., \& Schachtman, T. R. (1985). The several roles of context at the time of retrieval. In R. R. Miller \& N. E. Spear (Eds.), Information processing in animals: Conditioned inhibition (pp. 5188). Hillsdale, NJ: Erlbaum.

Pavlov, I. P. (1927). Conditioned reflexes. London: Oxford University Press.

Pearce, J. M., \& Hall, G. (1980). A model for Pavlovian learning: Variations in the effectiveness of conditioned but not of unconditioned stimuli. Psychological Review, 87, 532-552.

RaNDich, A., \& Ross, R. T. (1985). Contextual stimuli mediate the effects of pre- and postexposure to the unconditioned stimulus on conditioned suppression. In P. D. Balsam \& A. Tomie (Eds.), Context and learning (pp. 105-132). Hillsdale, NJ: Erlbaum.

Rescorla, R. A. (1981). Simultaneous associations. In P. Harzem \& M. D. Zeiler (Eds.), Predictability, correlation, and contiguity (pp. 47-80). Chichester, U.K.: Wiley.

Rescorla, R. A., \& Wagner, A. R. (1972). A theory of Pavlovian conditioning: Variations in the effectiveness of reinforcement and nonreinforcement. In A. H. Black \& W. F. Prokasy (Eds.), Classical conditioning II (pp. 64-99). New York: Appleton-Century-Crofts.

SChweitzer, L., \& Green, L. (1982). Reevaluation of things past. Pavlovian Journal of Biological Science, 17, 62-68.

Seraganian, P., \& VOM SAal, W. (1969). Blocking of the development of stimulus control when stimuli indicate periods of nonreinforcement. Journal of the Experimental Analysis of Behavior, 12, 767-772.

Speers, M. A., Gillan, D. J., \& Rescorla, R. A. (1980). Withincompound associations in a variety of compound conditioning procedures. Learning \& Motivation, 11, 135-149.

WAGNER, A. R. (1969). Stimulus validity and stimulus selection in associative learning. In N. J. Mackintosh \& W. K. Honig (Eds.), Fundamental issues in associative learning (pp. 90-122). Halifax, NS, Canada: Dalhousie University Press.

Wasserman, E. A., Carr, D. L. \& Deich, J. D. (1978). Association of conditioned stimuli during serial conditioning by pigeons. Animal Learning \& Behavior, 6, 52-56.

(Manuscript received July 2, 1986; revision accepted for publication December 3, 1986.) 